A number of distinguished guests made contributions to the discussion, the main additional speakers being Lord Rea, Louis Blom-Cooper and Martin Pick. The National Society for the Prevention of Cruelty to Children stressed that the mental health of the nation depends on its young and therefore child psychotherapy is not a luxury. Louis Blom-Cooper drew attention to the relevance of psychotherapists to the Children Act, in particular to ascertaining the wishes of children, and stated that Social Services and Health professionals needed to work in tandem. Both Lord Rea and Martin Pick felt that early treatment made sense from a long-term economic viewpoint as well as a mental health one.
It was felt to be essential to inform managers, NHS purchasers and providers, social services, education and voluntary groups of the relevance and importance of child psychotherapy. Emma Nicholson, MP, arrived with some solid proposals for influencing Government and it is hoped that the next step will be a report describing the nature and work of the profession, and making specific recommendations.

Finally the representative from the Royal College of Psychiatrists, Dr Nick Temple, lent support to the campaign and emphasised that the Royal College recognised child psychotherapists as valued, specialised and highly trained colleagues.

\title{
No twisted thought without a twisted molecule*
}

\author{
FranCES KLEMPERER, Senior Registrar, York Clinic, Guy's Hospital, London SE1 9RT
}

There can be no doubt that schizophrenia is associated with a variety of neurophysiological disturbances and disordered cognitive functioning. But we are only beginning to identify which brain areas are involved and to discover how abnormalities in these areas are related to abnormal psychological processes. This symposium, organised by Drs David and Cutting, offered an opportunity to learn about the work of both psychologists and psychiatrists in this field. Diverse research presented ranged from PET studies to the analysis of home videos, from epidemiological work to studies of cognitive performance.

Many of the issues addressed have concerned psychiatrists since 'schizophrenia' was first delineated. Does the term refer to a clearly distinguishable illness, or merely to a collection of symptoms? Professor Murray suggested that schizophrenia is a syndrome which may arise in adult life or be neurodevelopmental. Maternal influenza may be one important cause of the latter. But, in the view of Dr Goldberg, there is a lack of neurophysiological evidence for any subgroup, which argues in favour of considering schizophrenia as a single illness.

Can we speak of a 'primary' neurophysiological lesion? Dr Early related schizophrenic symptoms to PET results suggesting left striatopallidal hyperactivity. Dr Liddle had used PET to demonstrate underactivity in the prefrontal cortex in

* An International Symposium on 'The Neuropsychology of Schizophrenia', held at the Institute of Psychiatry, London 10-11 October 1991 . schizophrenics who have impaired ability to initiate activity. Drs Hemsley and Goldberg implicated the hippocampus in abnormal memory and integration of information. Dr Cutting proposed a right hemisphere dysfunction in schizophrenia.

Is there a basic cognitive deficit that underlies all of the symptoms of schizophrenia? Dr Frith discussed impairments in the schizophrenic patient's 'theory of mind', that is, the metarepresentation of one's own and others' beliefs and intentions. Dr Hemsley suggested that the basic disturbance is a failure to use redundant perceptual information in cognitive tasks. Dr Nuechterlein presented data on persistent abnormalities of sustained attention in schizophrenics, which may be an enduring indicator of schizophrenic vulnerability. An unusual and moving presentation from Dr Walker, using home videos, revealed early abnormalities in motor development and expression of affect in children who later developed schizophrenia.

There were many other excellent presentations. Different cognitive mechanisms were proposed for hallucinations, delusions and delusional misidentification syndrome. Abnormalities of memory and visual information processing, and the slowness of cognitive function in schizophrenia were also discussed.

This conference offered a comprehensive view, but no easy consensus of opinion. While advances are being made in integrating the 'bio' and the 'psycho' of schizophrenia, much remains to be discovered about both the disordered brain, and the disordered mind. 